\title{
Vom Feindbild zum differenzierten Europabild? Tschechien, Ostmitteleuropa und die europäische Integration
}

\author{
Vladimir Handl*
}

Der EU-Beitritt ist zutreffend als ein „glanzloser Arbeitserfolg von epochaler Bedeutung“ bezeichnet worden ${ }^{1}$ und wird eben so auch in den neuen Mitgliedstaaten wahrgenommen. Zwar ist die Euphorie der ,Rückkehr nach Europa' vorüber, die Normalität der EU-Mitgliedschaft wird jedoch allseits überwiegend positiv gewertet. Dieser Aufsatz befasst sich mit der Wahrnehmung der Europäischen Union in den Visegrád-Ländern, vor allem in der Tschechischen Republik. Um den historischen Kontext auszuleuchten werden nach einer kurzen Bestandsaufnahme der bisherigen Ergebnisse des EU-Beitritts jene Themen angesprochen, die in der offiziellen Politik des ,sozialistischen Lagers' die Wahrnehmung und Präsentation des Integrationsprozesses in den 1950er bis 1980er Jahren bestimmt haben. Beeinflussen sie in irgendeiner Weise auch heute noch den Diskurs über Europa? Die Sicht auf die Europäische Union ist differenzierter geworden, bleibt aber in der öffentlichen Meinung überwiegend positiv. Die Frage ist, wie hingegen die offensichtliche Polarisierung der politischen Eliten in manchen Staaten Ostmitteleuropas zu bewerten ist. Welche Folgen hat das Fehlen eines nationalen Konsens für die Wahrnehmung der Europäischen Union und für die Gestaltung der Europapolitik der betroffenen Länder?

\section{Der EU-Beitritt im Transformations- und Erweiterungskontext}

Die wirtschaftlichen Ergebnisse des EU-Beitritts sind überzeugend: Selbst Kritiker erkennen an, dass die Wachstumsraten im Jahr 2005 zwischen 3,2 Prozent in Polen und 10,2 Prozent in Lettland lagen, während in der EU-15 die Wirtschaft im Durchschnitt nur um 1,5 Prozent wuchs. Nahezu keine der EU-skeptischen Prognosen über die wirtschaftlichen Folgen des EU-Beitritts haben sich bestätigt. Auch die Situation auf dem Arbeitsmarkt, die Fiskal-, Währungs- und Lohnpolitik haben sich positiv entwickelt. ${ }^{2}$ Statt eines Beitrittschocks hat die Europäische Union den neuen Mitgliedstaaten zusätzliche ökonomische Impulse gegeben, die sich auch in wachsenden Handelsüberschüssen widerspiegeln. Gerade ursprüngliche Gegner des EU-Beitritts sind mit der Mitgliedschaft besonders zufrieden: So befürworten nun um die 70 Prozent der polnischen Bauern die Mitgliedschaft. ${ }^{3}$

* Dr. Vladimir Handl, Institut für Internationale Beziehungen, Prag.

Der Aufsatz basiert auf den Aktivitäten des Verfassers im Rahmen des Forschungsprogrammes des Instituts für internationale Beziehungen „Tschechische Republik in der internationalen Politik“ (VZ 4854 605401) und wurde in Grundzügen auf der wissenschaftlichen Konferenz „,50 Jahre Römische Verträge“ vorgetragen. Die Konferenz wurde vom Institut für Europäische Politik in Zusammenarbeit mit dem Arbeitskreis Europäische Integration und dem Centre International de Formation Européene in Berlin durchgeführt.

1 Barbara Lippert: Glanzloser Arbeitserfolg von epochaler Bedeutung: eine Bilanz der EU-Erweiterungspolitik 1989-2004, in: Barbara Lippert (Hrsg.): Bilanz und Folgeprobleme der EU-Erweiterung, Baden-Baden 2004, S. 13-72.

2 Ivan Okáli: How did the Trends in Economic Development of the New Member States Changed After Their EU-Accession, in: Milan Šikula/Veronika Hvozdíková (Hrsg.): Performance and Perspectives of the European Union as Seen by the New Member States. Post-Accession Monitoring Conference, Bratislava 2006, S. 76-81, hier S. 76.

3 Tomáš Pavlovský: Polsko, in: Vlastimil Havlík/Pater Kaniok (Hrsg.): Euroskepticismus a země střední a východní Evropy, Brno 2006, S. 45-60, hier S. 47. 
Im politischen Sinne sind die neuen Mitgliedstaaten zu Subjekten der Europapolitik geworden. Sie haben damit neue Möglichkeiten erhalten, an der Gestaltung der europäischen Politik mitzuwirken. Das legt allerdings nicht nur ihre Chancen, sondern auch ihre Schwächen offen. Vielfach sind die vielseitigen Asymmetrien zwischen den großen und kleinen beziehungsweise den alten und neuen Mitgliedsländern erst nach dem Beitritt deutlich geworden. Weniger die absolute Größe der einzelnen Länder spielt dabei eine wichtige Rolle, als vielmehr ihr jeweiliges institutionelles Potenzial und ihre Fähigkeit, aktiv eine kohärente und mehrheitsfähige Politik zu formulieren beziehungsweise sich an einer solchen zu beteiligen.

Die Referenden, die in allen Staaten Mittel- und Osteuropas (MOE) durchgeführt worden waren, bejahten ausnahmslos den EU-Beitritt - auch wenn die Haltung breiter Teile der Bevölkerung vor dem Beitritt Elemente einer „Europhobie“, 4 oder gar einer defensiven EuroAngst zeigten. Seit dem Beitritt hat sich die Haltung zur Europäischen Union differenziert entwickelt. Positive Einstellungen von 60 Prozent und mehr der Befragten sind in Rumänien, Polen und Slowenien anzutreffen, aber weniger als 50 Prozent in Tschechien (45 Prozent), Ungarn (41 Prozent) und Lettland (36 Prozent). Nur in der Frage der weiteren Erweiterung der Europäischen Union übertreffen alle Neumitglieder den EU-Durchschnitt von 49 Prozent. ${ }^{5}$ Die differenzierte Haltung gegenüber der Europäischen Union erklärt sich aus vielen Faktoren, die nicht unbedingt mit der aktuellen sozialen und wirtschaftlichen Lage zusammenhängen, sondern grundsätzlicher mit dem sozialen und politischen Kontext. Ähnliches gilt allerdings auch für die größten ,Skeptiker' der Europäischen Union - die Briten (35 Prozent), Österreicher (34 Prozent) und Finnen (29 Prozent). ${ }^{6}$

Auch in der Tschechischen Republik ist die Einstellung zur Europäischen Union von einer Vielzahl von Faktoren beeinflusst. Da der EU-Beitritt mit dem populären Slogan ,Rückkehr nach Europa' verbunden worden war, stellte er mehr als nur einen typischen ,topdown' Prozess dar. Denn die Wende von 1989 und die anschließende Westintegration fingen ja als ein ,bottom-up“ Prozess an, was die Übernahme des acquis communautaire erleichterte. ${ }^{7}$ Trotzdem führte der deutlich technokratische Charakter der Beitrittsvorbereitungen wie auch der Transformation zu einer weitgehenden Ent-Politisierung der EuropaStrategie. Durch die Einbindung in die Brüsseler Entscheidungsprozesse verstärkte der EUBeitritt in den neuen Mitgliedstaaten indirekt die ohnehin weit verbreitete Politikverdrossenheit. Die voranschreitende Individualisierung und schrittweise soziale Differenzierung der Gesellschaft wird nicht durch die Entfaltung der politischen Parteien und Zivilgesellschaft reflektiert. Mangelhaftes Vertrauen gegenüber und unter einzelnen politischen, institutionellen und wirtschaftlichen Akteuren wirkt sich negativ aus. Dagegen erscheinen die Europäische Union und ihre Organe als vertrauensvolle Institutionen. Der nationalen Regierung vertrauen nur 27 Prozent der Tschechen und gar nur 15 Prozent der Polen. Der Europäischen Kommission dagegen vertrauen 58 Prozent der Polen und 59 Prozent der Tschechen. ${ }^{8}$

4 Karen Henderson: Euroscepticism or Europhobia: Opposition attitudes to the EU in the Slovak Republic, Sussex European Institute, SEI Working Paper 50/2001, S. 13.

5 Europäische Kommission: Eurobarometer 67. Die öffentliche Meinung in der Europäischen Union. Erste Ergebnisse, Juni 2007, S. 24 und 32.

6 Europäische Kommission: Eurobarometer 67, 2007, S. 24.

7 Petr Drulák/Lucie Königová: The Czech Republic: from Socialist Past to Socialised Future, in: Trine Flockhart (Hrsg.): Socialising Democratic Norms. The Role of international Organisations for the Construction of Europe, Houndmills 2005, S. 149-168, hier S. 167.

8 Siehe die Analyse von Kai-Olaf Lang: Beitrittskater und posttransformative Depression. Ostmitteleuropas innenpolitische Wirrungen, SWP-Aktuell 19/2007, S. 5. 
Innerhalb der politischen Eliten gibt es solche, die ihre Gestaltungsmacht durch Europäisierung eingeschränkt sehen. Sie und weitere national gesinnte Akteure befürchten eine Verwässerung der nationalen Souveränität und Identität. Allgemein fehlt es an politischen Akteuren und Parteien, die politische Führung anbieten und öffentliche Unterstützung gewinnen können. Das führt dazu, dass in einigen Ländern Ostmitteleuropas im Endergebnis populistische und nationalistische Parteien Wahlgewinne erzielen, wie beispielsweise in Polen. ${ }^{9}$ In Tschechien führte dies während der Parlamentswahlen 2006 zu einer ideologischen Polarisierung der politischen Szene. In beiden Fällen wird die Europäische Union zu einem wichtigen Thema der Differenzierung der nationalen Politik, und trägt zur Herausbildung der parteipolitischen Positionen bei.

Dabei ist eine Differenzierung zwischen einer „,staatsnationalen“ und einer „,nationalföderativen“ Haltung zu erkennen. ${ }^{10}$ Die staatsnationale Haltung basiert auf der Souveränität der nationalen Staaten als dem zentralen Ordnungsprinzip und befürwortet ein zwischenstaatliches Modell der Europäischen Union. Die Position wird von euroskeptischen Parteien vertreten, ${ }^{11}$ in Tschechien also vor allem von der liberal-konservativen Občanská demokratická strana (ODS, Demokratische Bürgerpartei) und der linksradikalen Komunistická strana Čech a Moravy (KSČM, Kommunistische Partei Böhmens und Mährens). Die nationalföderative Haltung akzeptiert hingegen, wo notwendig, überstaatliche Elemente des Integrationsmodells; auf der tschechischen politischen Szene ist sie vor allem durch die sozialdemokratische Česká strana sociálnì democratická (ČSSD, Tschechische Sozialdemokratische Partei), die Grünen sowie die christlichen Demokraten Křest'anská a demokratická unie - Československá strana lidová (KDU-ČSL, Christliche und Demokratische Union-Tschechische Volkspartei) vertreten. ${ }^{12}$

Beide Strömungen sind in allen neuen Mitgliedstaaten Ostmitteleuropas langfristig vertreten. Vor dem EU-Beitritt hatte diese Trennlinie nur einen begrenzten Einfluss. Der ,permissive Konsens" in Fragen der Europapolitik war in der Tschechischen Republik ebenso wie in Polen allein auf den Beitritt zur Europäischen Union und zur NATO begrenzt. Dieser Konsens ist nun effektiv aufgebraucht worden, und die unterschiedlichen Positionen verursachen erhebliche Schwankungen in der Ausrichtung der tschechischen und auch polnischen Europapolitik. Ähnliches kann in Ungarn erwartet werden, falls der bürgerlich-konservative FIDESZ die nächsten Wahlen gewinnt.

\section{Die Europäische Union einst und heute: Wandel des Europabildes?}

Vor dem Jahre 1989 entwickelten sich die Beziehungen der Ostblockstaaten zum westeuropäischen Integrationsprozess in drei Phasen: bis Anfang der 1970er wurden die Europäischen Gemeinschaften feindselig betrachtet und nicht offiziell anerkannt. Während einer Zwischenphase (1972-1984) wuchs ein begrenztes Interesse an einer Zusammenarbeit, die dann in der dritten Phase (zweite Hälfte der 1980er) langsam realisiert wurde. ${ }^{13}$ Das belegte etwa die indirekte Anerkennung der Europäischen Gemeinschaften seitens des General-

9 Vgl. Lang: Beitrittskater und posttransformative Depression, 2007, S. 5-6.

10 Für diese Unterteilung der politischen Klasse bezüglich der europäischen Integration und eine entsprechende Analyse der tschechischen Politik siehe Volker Weichsel: Tschechien in Europa. Nationalpolitische Traditionen und integrationspolitische Konzepte, Berlin 2007.

11 Für das Konzept des parteibezogenen Euroskeptizismus siehe Aleks Szczerbiak/Paul Taggart: Theorizing Party-Based Euroscepticism: Problems of Definition, Measurement and Causality, Sussex European Institute, SEI Working Paper 96/2003.

12 Weichsel: Tschechien in Europa, 2007, S. 233-254.

13 Jan Karlas/Petr Kratochvíl: Czechoslovakia/Czech Republic and European Integration: During and After the Cold War, in: Journal of European Integration History 2/2004, S. 25-42, hier S. 28. 
sekretärs der Kommunistischen Partei der Union der Sozialistischen Sowjetrepubliken, Leonid Brezhnew, im Jahre 1972.

Während es in der politischen Praxis zu einer indirekten Anerkennung der Europäischen Gemeinschaften kam, wurden sie in der politischen und ideologischen Auseinandersetzung bis in die späten 1980er Jahre nahezu ignoriert, als ob die Doktrin des Marxismus-Leninismus sie nicht einzuordnen verstünde. Das ideologisierte Verständnis der internationalen Beziehungen des Zentralkomitees der Kommunistischen Partei der Sowjetunion (KPdSU) arbeitete mit einem integrierten Feindbild, das sich auf die amerikanische Hegemonie des Westens konzentrierte. In seinem Rechenschaftsbericht auf dem XXIV. Parteitag der KPdSU im Jahre 1981 sprach Leonid Brezhnew ausschließlich über die USA und die NATO sowie die bilateralen Beziehungen mit den einzelnen westlichen Ländern - aber die Europäischen Gemeinschaften blieben unerwähnt. ${ }^{14}$ Ebenso erwähnte Generalsekretär Gorbatschow die Europäischen Gemeinschaften auf dem XXVII. Parteitag (1986) in seiner Rede nur einmal - und zwar als einen verfehlten Versuch, die ,innerimperialistischen Gegensätze" zu mildern. ${ }^{15}$

Nach ,1989‘ fand zunächst eine Idealisierung der Europäischen Union statt. Es dominierte eine ,euphorische pro-EU Haltung', die schon Mitte der 1990er Jahre in eine realistischere und bisweilen auch euroskeptische Haltung überging, wobei nationale Interessen und Sorgen um die Bewahrung nationaler Identität akzentuiert wurden. ${ }^{16}$ Sehr bald zeigte sich auch, dass die Palette der kritischen Argumente, die in den Jahren 1957-1989 seitens der Führung der Ostblockstaaten benutzt wurden, in einer gewandelten Form wieder in der Debatte auftauchten.

\section{Das Klassenargument und das soziale Argument}

Die ursprüngliche Kritik berief sich auf Lenins These, die „Vereinigten Staaten von Europa“ seien auf kapitalistischer Basis ,entweder unmöglich oder reaktionär". ${ }^{17}$ Auch wenn in den 1970er und 1980er Jahren die Integrationsfähigkeit Westeuropas positiv bewertet wurde, war Lenins Einschätzung als gültig betrachtet worden, soweit es um den „Klasseninhalt imperialistischer Europapolitik" ging. ${ }^{18}$ Die Europäischen Gemeinschaften wurden als ein Instrument des Monopolkapitals zur Verteidigung der Klassen- und Kolonialherrschaft sowie den Kampf gegen die „Staaten der sozialistischen Gemeinschaft“ dargestellt. ${ }^{19}$

Außerhalb der orthodoxen Parteisekretariate wurde diese Sicht der Europäischen Gemeinschaften jedoch schon lange vor dem Jahr 1989 relativiert. In der Öffentlichkeit Ostmitteleuropas entwickelte sich ein überwiegend positives Bild der Europäischen Gemeinschaften als einer Wohlfahrtsgemeinschaft. Gegenüber der sozialistischen Mangelwirtschaft bis 1989 und den nachfolgenden Schwierigkeiten im Transformationsprozess wurde die wirtschaftliche und soziale Attraktivität der Europäische Gemeinschaften/Europäischen Union betont. Es war fast unumgänglich, dass die sozialen und ökonomischen Motive bei

14 Zpráv a UV KSSS a další úkoly strany ve vnitřní a zahraniční politice, in: XXVI. Sjezd KSSS. Dokumenty a materiály, Praha 1981, S. 25.

15 Politická zpráva ÚV KSSS, 25.02.1986, in: XXVII sjezd KSSS, 25.02-06.03.1986, Dokumenty a materiály, Praha 1986, S. 16.

16 Vgl. Rudolf Hrbek: The 2004 EP Elections in the ten new EU Member States and the Enlargement of the European Party System - an Introduction, in: Rudolf Hrbek (Hrsg.): European Parliament Elections 2004 in the Ten New EU Member States. Towards the Future European Party System, Baden-Baden 2005, S. 20.

17 Wladimir I. Lenin: Über die Losung der Vereinigten Staaten von Europa, Berlin 1968, S. 343, zitiert nach Wilhelm Ersil (Hrsg.): Westeuropa. Politische und militärische Integration, Berlin 1985, S. 18.

18 Ersil (Hrsg.): Westeuropa. Politische und militärische Integration, 1985, S. 18.

19 История международных отношений и внешней политики СССР. Москва 1987, Bd. 2, S. 264-275. 
der Neuorientierung auf die Europäische Union prioritär waren. ${ }^{20}$ Selbst in der Slowakei, die unter Vladimir Mečiars Regierung den Anschluss an die anderen Visegrád-Länder in der Integrationsanstrengung verloren hatte, überwogen die materiellen Motive andere Begründungen wie etwa die „Demokratie in der Slowakei zu stärken“ und die „Integration in die westliche Welt voranzutreiben“.21

Bis heute bleibt das ,klassische' Klassenargument zentral für die radikale Linke (so die Tschechische Kommunistische Partei Böhmens und Mährens) und zum Teil auch für die extreme Rechte Sdružení pro republiku - Republikánská strana Českolovenska (SPR-RSČ, die außerparlamentarische Partei der tschechischen Republikaner): Die entschiedenen Gegner des Kapitalismus und des Westens an beiden Rändern des politischen Spektrums lehnten den EU-Beitritt als endgültige Sanktionierung der Herrschaft des Kapitals ab.

In der sozial-demokratischen Linken und den Gewerkschaften ist der marxistische ,Arbeit-Kapital-Gegensatz ' bei den Themen ,soziale Sicherheit", ,Solidarität" und ,Kohäsion " hoch präsent. Die soziale Dimension der Transformation und der EU-Integration wird auch von sozial gesinnten Vertretern der politischen Mitte zum Thema gemacht - am stärksten von den christlich-demokratischen Parteien. Die moderaten Kräfte betrachten - anders als die Mehrheit der radikalen Linken - den EU-Beitritt grundsätzlich als einen Teil der Lösung, nicht des Problems: Das soziale Model der Europäischen Union besitzt weiterhin eine große Attraktivität. ${ }^{22}$ In letzter Zeit scheint jedoch der Prozess der wirtschaftlichen Liberalisierung und der Globalisierungsdruck der Frage der wachsenden Ungleichheit und daraus folgenden Ungerechtigkeit neue politische Relevanz (und Brisanz) zu verleihen. ${ }^{23}$ Die neue sozial-ökonomische Konstellation belebt das Thema Arbeit-Kapital in seiner eher traditionellen Form des Klassengegensatzes. Das Thema bleibt relevant, solange die Unterschiede zwischen den Gewinnern und den Verlierern weiter wachsen und die negativ betroffenen Schichten immer breiter werden. Es ist zu erwarten, dass damit auch der europapolitische Diskurs in der Tschechischen Republik neue Akzente bekommen wird.

\section{Das geopolitische und geostrategische Argument}

In der Politik des Ostblocks verband sich die Klassensicht ständig mit dem geostrategischen Argument. Man betrachtete den westeuropäischen Integrationsprozess als „ökonomische Stütze der NATO“ und als ,,wichtigen Faktor der Politik der Stärke“, der ,der militärisch-politischen Konfrontation mit den sozialistischen Staaten untergeordnet" wurde. ${ }^{24}$ Die Europäischen Gemeinschaften waren aus dieser Sicht primär eine ,wirtschaftliche Untermauerung des europäischen Flügels der NATO“. ${ }^{25}$

Man nahm an, im Westen entstünde eine ähnlich hierarchische Struktur, wie im Osten Europas. Diese Einschätzung wurde allerdings schon Mitte der 1970er Jahre korrigiert: Die Europäischen Gemeinschaften seien zu einem ,zweiten Zentrum des imperialistischen Weltsystems geworden“, das sich von der ,amerikanischen Vormundschaft“" schrittweise befreie. ${ }^{26}$ Die ursprüngliche Verdammung der Europäischen Gemeinschaften wich dem

20 Bálint Bala: Sociology of the European Union - Past and Present, in: Ilja Srubar (Hrsg.): Problems and Chances of the East Enlargement of the EU, Hamburg 2003, S. 13-28, hier S. 22.

21 Vladimír Krivý: Slovakia and the EU, in: Srubar (Hrsg.): Problems and Chances of the East Enlargement of the EU, 2003, S. 10-112, hier S. 108.

22 Vgl. Brigita Schmögnerová: The European Social Model: Reconstruction or Deconstruction? A View from a Newcomer, Friedrich-Ebert-Stiftung, Bonn 2005.

23 Vgl. die Analyse von Petra Böhnke: Poverty and Social Integration in the Enlarged Europe, WZB Discussion Paper SP 2007-203, Berlin 2007.

24 Ersil (Hrsg.): Westeuropa. Politische und militärische Integration, 1985, S. 49-50.

25 Vladimír Soják: Mezinárodní vztahy naší doby, Praha 1976, S. 59-60. 
Interesse, den Integrationsprozess als einen Prozess der Verselbstständigung der westeuropäischen Länder gegenüber den USA zu unterstützen. ${ }^{27}$

Daran knüpfte die geostrategische Diskussion nach 1989 an. Das Ende des Kalten Krieges ,befreite` die Europäischen Gemeinschaften/Europäische Union von dem bipolaren Gegensatz. Alle MOE-Staaten, die zugleich neue NATO-Staaten sind, reagieren bei sicherheitspolitischen Themen reflexartig, atlantisch‘. Diese Einschätzung gilt offensichtlich vornehmlich für die politischen Eliten und betrifft die Öffentlichkeit Ostmitteleuropas nur mit gewissem Vorbehalt; die Vorreiter des Atlantizismus in der Europäischen Union sind weiterhin die Niederländer, Briten, Italiener und die Deutschen. ${ }^{28}$

Trotzdem bleibt die Tendenz stark, die Atlantische Allianz und die strategische Anbindung an die USA als Garantie gegen alle möglichen Bedrohungen wahrzunehmen - inklusive einer Wiederherstellung der Großmachtbestrebungen Russlands oder einer Neuauflage der deutschen Hegemonie. Die NATO, nicht die Europäische Union, wird als die Sicherheitsgemeinschaft betrachtet. Einige hard-core Atlantiker stellen selbst die verbreitete Sichtweise der Europäischen Union als einer Friedensgemeinschaft in Frage. ${ }^{29}$ Ein Streitpunkt sind vor allem Ansätze für eine autonome europäische Sicherheits- und Verteidigungspolitik. Die Atlantiker in den neuen wie auch den alten EU-Mitgliedstaaten fürchten eine weitere Entfremdung auf beiden Seiten des Atlantiks und damit eine Abschwächung der strategischen (Selbst-)Verpflichtung der USA gegenüber der Europäischen Union.

Das Störpotenzial dieses Themenkomplexes blieb während der 1990er Jahre begrenzt, da die sicherheitspolitische Relevanz der Europäischen Union beschränkt war. Außerdem wurde das Sicherheitsbedürfnis der MOE-Staaten mit dem Beitritt zur NATO weitgehend befriedigt, sodass etwa in Polen der EU-Beitritt als weniger dringlich angesehen wurde. ${ }^{30}$ Allmählich gewann jedoch das geostrategische Argument an Gewicht. Mit den NATO-Luftangriffen gegen Jugoslawien und später mit dem problematischen Irak-Krieg bekam der ,Europäismus‘ derjenigen Mittel- und Osteuropäer einen starken Impuls, die Gewaltanwendung skeptisch oder ablehnend betrachten und/oder antiamerikanische Reflexe haben. Entsprechend argumentieren seit 1999 die Euro-Aktivisten in der tschechischen Linken - inklusive mancher Kommunisten - Ziel müsse es sein, Europa zu einem eigenständigen Akteur oder - in radikaler Variante - sogar zu einem Gegengewicht zu den USA zu machen.

Selbst Mainstream-Atlantiker sind in der Frage der europäischen Verteidigungspolitik nicht einig. In Polen, wo die Verwicklung in den Irak-Krieg an der Seite der Amerikaner nicht nur sicherheitspolitisch umstritten blieb, sondern auch keine wirtschaftliche Vorteile brachte, wurde das Interesse der polnischen Politik an der europäischen Sicherheits- und Verteidigungspolitik stimuliert. ${ }^{31}$ Selbst Premierminister Kaczynski, der den Bindungen an die USA große Aufmerksamkeit widmete, wurde zu einem Befürworter der Idee einer Europäischen Armee. ${ }^{32}$ Tschechische Atlantiker bleiben allerdings gegenüber einer funktionsfähigen Europäischen Sicherheits- und Verteidigungspolitik skeptisch.

26 Soják: Mezinárodní vztahy naší doby, 1976, S. 60.

27 Дипломатический словарь. Москва 1985, Bd. I, S. 353.

28 Pierangelo Isernia/Philip P. Everts: European Public Opinion on Security Issues, in: European Security 4/2006, S. 451-469, hier S. 456-457.

29 Lukáš Petř́ik: Je EU př́íčinou míru v Evropě?, in: CEP Newsletter 8/2007, S. 8, abrufbar unter: http://cepin.cz/ docs/newslettery/2007-08.pdf (letzter Zugriff: 10.09.2007).

30 Zdzislaw Krasnodębski: Poland and the European Union: Changing Attitudes, in: Srubar (Hrsg.): Problems and Chances of the East Enlargement of the EU, 2003, S. 87-99, hier S. 90.

31 Marcin Zaborowski: From America's Protégé to Constructive European. Polish Security Policy in the TwentyFirst Century, ISS Occasional Paper 56/2004.

32 Institut für Europäische Politik (Hrsg.): EU-25/27 Watch, No. 4, January 2007, Berlin, S. 92. 
Insgesamt hat der Irak-Krieg den ,atlantischen Reflex ' abgeschwächt - das Projekt der amerikanischen Raketenabwehr hat ihn allerdings wieder aufleben lassen. Die Frage bleibt, ob und inwieweit die Debatte über die Raketenabwehr die innere Kohärenz der Europäischen Union beeinträchtigen wird. Eine kooperative euro-atlantische Lösung wäre für die Entwicklung der Europäischen Union als einer Sicherheitsgemeinschaft (im Sinne einer funktionsfähigen europäischen Zivilmacht) sehr hilfreich. Die eindeutige Anlehnung an die US-Garantiemacht und damit an eine militärische Antwort auf die neuen Sicherheitsgefährdungen spiegelt Zweifel der tschechischen und polnischen hard-core Atlantiker nicht nur an der Europäischen Union, sondern auch an der Handlungsfähigkeit der erweiterten NATO wider.

\section{Politische Kultur und Ideologie}

Auch nach dem Jahr 1989 blieb Kritik am Liberalismus des Westens ein Teil der antiwestlichen Argumentation. Mit der Öffnung gegenüber dem Westen kam auch eine „Flut negativer Nebeneffekte der Zivilisationsentwicklung“, die ,,antiwestliche Phobien“ hervorriefen. ${ }^{33}$ Vor allem der polnische Sender ,Radio Maryja ‘ und die Partei ,Liga der Polnischen Familien“ (Liga Polskich Rodzin, LPR) machten die Kritik am westlichen Lebensstil zum Kern ihrer polnischen Identität.

Auch Teile der slowakischen konservativen christlich demokratischen Krest'anskodemokratické Hnutie (KDH, Christlich-demokratische Bewegung) grenzten sich von angeblich typischen Erscheinungsformen Westeuropas wie Konsumdenken, Hedonismus, Homosexualität, Abtreibungen und Glaubensschwäche und Glaubensverlust der Christen ab. ${ }^{34}$ Sie wendeten sich gegen den Europäischen Verfassungsvertrag und forderten die Bewahrung der ,nationalen Identität im kulturell-ethischen Bereich“. ${ }^{35}$ Die anti-liberalen kulturellen Strömungen geraten damit in die Nähe der anti-liberalen/anti-westlichen kommunistischen Linken in Tschechien oder der Slowakei.

Viel größer sind jedoch die Unterschiede in der politischen Kultur der EU-15 und der der neuen Mitgliedsländer. Die Funktionsweise der Europäischen Union als einer Konsensgemeinschaft, die auf allseitig akzeptierte Kompromisse baut, ist der politischen Kultur der MOE-Länder recht fremd. Die Nachkriegsentwicklung im Osten Europas schwächte die Ansätze einer politischen Kultur der Kompromisssuche deutlich ab. Die Monopolstellung der kommunistischen Parteien verstärkte die Tendenz, Politik als einen Kampf zwischen unvereinbaren Gegensätzen zu betrachten. Auch ein Multilateralismus, der auf das Zusammenlegen von Souveränität hinausläuft, konnte sich in der Region kaum entwickeln. Die strikt hierarchische Struktur des Ostblocks basierte ja auf Unterordnung der Satelliten unter das Moskauer Zentrum und förderte keine horizontalen Bindungen. Nationale Interessen, Statusfragen und Prestige spielen in der Zeit nach 1989 also eine wichtige Rolle. Daher die Schwierigkeiten der Neumitglieder bei der Kompromisssuche auf EU-Ebene. So kommen neuere Analysen zu der Schlussfolgerung, in der ungarischen Politik herrsche die Tendenz vor, jeden Kompromiss als Niederlage und Schwäche zu interpretieren. ${ }^{36}$ Ähnliches gilt ge-

33 Anna Wolf-Powenska: Identität in der Wendezeit, in: Bohdan Kozsel/Hans Maretzki (Hrsg.): Länder Mittelund Südosteuropas auf der Suche nach neuer Identität, Potsdam 1998, S. 27-40, hier S. 33.

34 Eva Lešková: Slovensko, in: Havlík/Kaniok (Hrsg.): Euroskepticismus a země stř̌ední a východní Evropy, 2006, S. 31-44, hier S. 37.

35 Vladimír Leška: Změny na slovenské politické scéně a jejich vliv na česko-slovenské vztahy, in: Mezinárodní politika 9/2004, S. 18-21, hier S. 18-19, abrufbar unter: http://www.iir.cz/upload/MP/MPArchive/2004/ MP092004.pdf (letzter Zugriff: 23.9.2007). 
nerell für staatsnational orientierte Akteure, wie der Kampf der polnischen und tschechischen Euroskeptiker gegen den Vertrag über eine Verfassung für Europa demonstrierte.

Andere Analysen zeigen, dass Unterschiede zwischen der EU-15 und den Neumitgliedern in Bezug auf das Modernisierungsniveau, politische Kultur oder Machtpotenzial im Ergebnis das Vertrauensverhältnis innerhalb der Europäischen Union abschwächen. ${ }^{37}$ Es ist eindeutig, dass die Heterogenität der Europäischen Union mit der Osterweiterung zugenommen hat. Die Öffentlichkeit der neuen Mitgliedstaaten befindet sich allerdings - wenigstens bei einer generalisierenden Betrachtung - sehr nahe bei den in der EU-15 prioritären politischen und sozialen Werten. ${ }^{38}$ Die Europäische Union als Gemeinschaft der postmaterialistischen und individuellen Werte, für die nationale Souveränität keine unantastbare Größe darstellt, ist allerdings nicht akzeptabel für die staatnational orientierten Eliten. Eine prominente Stimme ist der tschechische Präsident Václav Klaus. Er wendet sich gegen jegliche ,-ismen “ in der Politik, und lehnt sie entschieden als Gefährdung der Freiheit ab: „Da gibt es zum Beispiel den Sozialdemokratismus, der nichts anderes ist als eine weichgespülte Form des Kommunismus, oder den Menschenrechtismus, der auf der Idee meist positiver, global durchzusetzender Rechte beruht. Dann haben wir den Internationalismus, Multikulturalismus, Feminismus, Europäismus und Ökologismus nebst einer ganzen Rüstkammer ähnlicher Dogmen und Ideologien. Der Kommunismus ist passé, aber der Drang zum Topdown-Regieren begleitet uns weiterhin - oder vielleicht wieder.“39

\section{Die konstitutionellen Argumente}

Vor allem in den 1950er und 1960er Jahren wurden die Europäischen Gemeinschaften als undemokratisch dargestellt, während der Rat für Gegenseitige Wirtschaftshilfe (RGW) als eine neuartige, wahrhaft demokratische Organisation präsentiert wurde, da sie nach dem Prinzip der Einstimmigkeit funktionierte. ${ }^{40}$ Unter möglichen Integrationsmodellen für Westeuropa zog die östliche Doktrin der internationalen Beziehungen eindeutig das konföderative Modell (und damit die französische Politik) dem als konservativ und undemokratisch dargestellten föderativen Modell vor. Die Europapolitik der Bundesrepublik Deutschland wurde explizit kritisch betrachtet. ${ }^{41}$

Anfang der 1990er Jahre orientierten sich die MOE-Staaten bei ihrer Suche nach einem neuen Leitbild auf den Westen und insbesondere die Europäische Union. Allerdings überlebte eine gewisse Zurückhaltung bezüglich der supranationalen Elemente der institutionellen Entwicklung. Einen Testfall der konstitutionellen Politik der jeweiligen Regierungen stellt ihre Position gegenüber dem Verfassungsvertrag für Europa dar. Während des Konvents haben die MOE-Staaten tendenziell die Bewahrung ihrer jeweiligen Positionen im institutionellen Gefüge der Europäischen Union verfolgt. Nur zwei Regierungen haben sich während der Ratifikationsphase gegen den Europäischen Verfassungsvertrag ausgesprochen: die tschechische und die polnische, in denen euroskeptische Parteien eine führende

36 Daniel Stankov: Mad'arsko, in: Havlík/Kaniok (Hrsg.): Euroskepticismus a země střední a východní Evropy, 2006, S. 83-96, hier S. 83-84.

37 Jan Delhey: Do Enlargements Make the European Union Less Cohesive? An Analysis of Trust Between EU Nationalities, in: Journal of Common Market Studies 2/2007, S. 253-279.

38 Europäische Kommission: Special Eurobarometer 225. Social values, Science and Technology, Juni 2005, S. 98-99.

39 Václav Klaus: Sozialdemokratismus, Europäismus, Ökologismus. Populäre Ismen und der Drang zum Regieren von oben herab, in: Schweizer Monatshefte, 30.8.2007, abrufbar unter: http://www.klaus.cz/klaus2/asp/clanek.asp?id=OOvKP1imaJfv, (letzter Zugriff: 10.09.2007).

40 История международных отношений и внешней политики СССР, 1987, Bd. 2, S. 24.

41 История международных отношений и внешней политики СССР, 1987, Bd. 3, S. 51-58. 
Rolle spielten. In beiden Fällen haben sich euroskeptische Kreise weit von der eigenen Bevölkerung entfernt: Eine große Mehrheit der polnischen Öffentlichkeit (68 Prozent) sah im Jahre 2006 einen Bedarf für eine ,europäische Verfassung ‘ und unterstützte die Vertiefung der Zusammenarbeit in der Europäischen Union (69 Prozent); gleichzeitig forderten allerdings 57 Prozent die maximale Beibehaltung der nationalen Souveränität. ${ }^{42}$ Auch die tschechische Bevölkerung unterstütze die weitere Vertiefung der Europäischen Union und bejahte zu 56 Prozent den Verfassungsvertrag. ${ }^{43}$

Ein Erklärungsversuch für die Spaltung der politischen Elite in der Frage der zukünftigen Gestalt der Europäischen Union weist auf historisch gewachsene ,reaktive Identitätsfaktoren'. Der Kampf gegen hegemoniale Mächte, aber auch die Kooperation mit ihnen führten in der Vergangenheit immer wieder zu einer Spaltung der nationalen Eliten in das Lager der Akzeptanz und das der Ablehnung. Entsprechend ist die gesellschaftliche Elite in Bezug auf die Europäische Union in zwei Lager geteilt: das eine identifiziert sich hauptsächlich mit der Europäischen Union, das andere befindet sich in Konfrontation zu ihr. ${ }^{44}$

Ein anderer Grund für die schwierige Positionierung der politischen Eliten der ostmitteleuropäischen Länder besteht darin, dass der EU-Beitritt ein Teil des Emanzipationsprozesses dieser Länder war. Die Emanzipation von der sowjetischen Hegemonie machte sie zu Trägern der Tradition einer modernen nationalen Staatlichkeit. Die staatsnational orientierten Parteien und Kreise tendieren dazu, den zentralistischen Nationalstaat als einen Wert an sich zu betrachten. ${ }^{45}$ Für sie stellt der Nationalstaat zugleich eine Schutzbarriere gegen übermäßige Eingriffe in den einzig legitimen politischen Prozess - also in die nationale Gestaltung der Politik - dar. Aus ihrer Sicht bleibt hingegen die Identität der Europäischen Union schwach und die Identifizierung mit dem Nationalstaat und der Region zeigt sich weiterhin als sehr viel vitaler. ${ }^{46}$

Die euroskeptischen Parteien, die gegen eine weitere Vertiefung der Europäischen Union auftreten, sind zu einem wichtigen Bestandteil der Parteiensysteme und der politischen Landschaft insgesamt geworden. Stellen sie die Regierung oder sind sie daran beteiligt, können sie - wie es die polnische Regierung klar demonstrierte - den Entscheidungsprozess in der Europäischen Union beinahe zum Stillstand bringen. Für die Euroskeptiker, oder, wie sie sich selbst sehen, die Eurorealisten, ist auch die Akzeptanz der ,Methode Monnet " problematisch. Die ,konstruktive Mehrdeutigkeit“47 des Integrationsprojektes wird im Kontext des Spill-over-Effektes von den staatsnational orientierten Akteuren als bedrohlich wahrgenommen. Wenn man die Schreckensvision der Euroskeptiker beschreiben will, kann man als eine Definition der Methode Monnet benutzen: „Was ,klein“ anfängt, wird einer internen, endogenen Logik folgend zu einem noch nicht eindeutig festlegbaren Ziel vorstoßen“" ${ }^{48}$ Die

42 The Social Perception of the Results of Poland's EU Membership, Institute of Public Affairs, Policy Paper 2006, abrufbar unter: http://www.isp.org.pl/files/20994854830540572001150709169.pdf, S. 16-17. (letzter Zugriff: 10.09.2007).

43 Europäische Kommission: Eurobarometer 66. Die öffentliche Meinung in der Europäischen Union. Erste Ergebnisse, Dezember 2006, S. 204.

44 Für dieses Argument siehe Raivo Vetik/Gerli Nimmerfelft/Marti Taru: Reactive Identity versus EU Integration, in: Journal of Common Market Studies 5/2006, S. 1079-1102.

45 So unterstützte z.B. das politische Programm der ODS den EU-Beitritt, lehnte es allerdings ab, den tschechischen Staat in supranationalen Strukturen zu verwässern. Siehe Politicky program ODS, Praha 02.11.1995.

46 Siehe dazu die Analyse von Bettina Thalmeier: Möglichkeiten und Grenzen einer Identitätspolitik, CAP Analyse 6/2006.

47 Heinrich Schneider: Ein Wandel europapolitischer Grundverständnisse? Grundsatzüberlegungen, Erklärungsansätze und Konsequenzen für die politische Bildungsarbeit, in: Mathias Jopp/Andreas Maurer/Heinrich Schneider (Hrsg.): Europapolitische Grundverständnisse im Wandel. Analysen und Konsequenzen für die politische Bildung, Bonn 1998, S. 19-148, hier S. 41. 
Entwicklung der europäischen Integration seit 1957 unterteilt beispielsweise der tschechische Präsident Klaus in zwei Perioden: die erste wird als Liberalisierungsphase charakterisiert und findet seine Unterstützung: die Europäischen Gemeinschaften entwickelten sich auf der Grundlage der Regierungszusammenarbeit und bauten die (Zoll-)Barrieren zwischen den Mitgliedstaaten der Europäischen Gemeinschaften ab. Die zweite Periode begann mit dem Maastrichter Vertrag und wird als Phase der Harmonisierung und supranationalen Entwicklung der Europäischen Union aufgefasst, die aus Sicht von Klaus eine unakzeptable Zentralisierung, Top-down-Überregulierung und Homogenisierung des gesellschaftlichen Lebens mit sich brachte. ${ }^{49}$ Er glaubt, es entstünde so ein ,post-gouvernementaler Totalitarismus von Eliten, die vom Integrationsprozess profitieren“.50 Aus dieser Sicht ist eine Europäische Union mehrerer Geschwindigkeiten die richtige Antwort auf die innere Heterogenität der Europäischen Union. Anders als die national-konservative Regierung Polens, die während der Verhandlungen über den Verfassungsvertrag im Juni 2007 eine ,Kamikaze-Politik betrieb, zeigte die tschechische Regierung gegen Ende Flexibilität und trat während des Gipfeltreffens im Juni 2007 lediglich einer unkontrollierbaren Übertragung von Kompetenzen auf die europäische Ebene, zum Beispiel mit Hilfe von Passarelle-Regelungen, entgegen. Damit entfernte sie sich deutlich von den Positionen, die Präsident Klaus einnimmt.

\section{Funktionsfähigkeit und Good Governance der Europäischen Union}

Die skeptische Wahrnehmung der Funktionsfähigkeit der Europäischen Gemeinschaften wich schon Mitte der 1960er Jahre einer impliziten Anerkennung. Es wurden Befürchtungen geäußert, die Europäischen Gemeinschaften könnten als Handelsblock die östlichen Länder diskriminieren. ${ }^{51}$ Ende der 1970er Jahre begann man, über den objektiven und dauerhaften Charakter der ökonomischen Integration Westeuropas zu sprechen. ${ }^{52}$ Zugleich beschuldigte man die Europäischen Gemeinschaften, sie wollten ihre ökonomische Stärke ausnützen um den Ostblock zu spalten und bilateral differenzierte Beziehungen mit ausgewählten Ländern zu entwickeln. ${ }^{53}$ Während der 1980er Jahre schlossen einzelne Ostblockstaaten bilaterale Sektoral- und Handelsabkommen mit den Europäischen Gemeinschaften; der RGW und die Europäischen Gemeinschaften unterzeichneten schließlich im Juni 1988 eine ,gemeinsame Erklärung' 54

Die neue politische und wirtschaftliche Elite, die mit der Wende im Jahre 1989 die Führung in einzelnen MOE-Ländern übernommen hat, sah die Europäische Union als Ziel und zum großen Teil auch als ein Modell für ihre Politik. Es gab jedoch auch kritische Stimmen:

48 Wolfgang Wessels: Jean Monnet - Mensch und Methode. Überschätzt oder Überholt?, Reihe Politikwissenschaft Nr. 74, Wien 2001, S. 8, abrufbar unter: http://aei.pitt.edu/280/01/pw_74.pdf (letzter Zugriff: 10.09.2007).

49 Václav Klaus: Some Doubts about the EU's Ever-Closer Future, The Bridge - Forum Dialogue, Luxembourg, 08.03.2006, abrufbar unter: http://www.klaus.cz/klaus2/asp/clanek.asp?id=WpNHn7MwQdlA (letzter Zugriff: 10.09.2007).

50 Věra Řiháčková: Die gegenwärtige Einstellung der politischen Kräfte in der Tschechischen Republik zum Vertrag über eine Verfassung für Europa. Analysen aus der Tschechischen Republik, Friedrich-Ebert-Stiftung, Prag 1/2007, S. 4. Für eine ähnliche Analyse vlg. Vladimír Handl: Tschechische Europapolitik: schwierige Positionierung während der deutschen Ratspräsidentschaft. Analysen aus der Tschechischen Republik, FriedrichEbert-Stiftung, Prag 3/2007. Beide Publikationen abrufbar unter: http://www.fesprag.cz/publikationen.php (letzter Zugriff: 24.9.2007).

51 Václav Kotyk: O zahraniční politice socialistických států, Praha 1963, S. 80-81.

52 Jewgenij Primakow bezeichnete es als einen wichtigen Erfolg des Instituts für Weltwirtschaft und Internationale Beziehungen, IMEMO, dass die europäische Integration als ein Thema erstmals überhaupt öffentlich diskutiert werden konnte. Vgl. Евгений Примаков: Минное поле политики, Москва 2007, S. 33.

53 История международных отношений и внешней политики СССР, 1987, Bd. 3, S. 65-69.

54 Vgl. Barbara Lippert: Etappen der EG-Osteuropapolitik: Distanz - Kooperation - Assoziierung, in: integration 3/1990, S. 111-125. 
Neoliberale Ökonomen und liberal-konservative Parteien hätten die Europäischen Gemeinschaften gerne auf eine Freihandelszone reduziert. Der freie Wettbewerb und die vier Freiheiten, die in den Römischen Verträgen definiert worden waren, stellten für sie den Kern des Integrationsprozesses einer liberalen Wirtschaftsgemeinschaft dar. Unbestritten ist auch die Funktion der Europäischen Union als einer Stabilitätsgemeinschaft. Auch manche liberale Ökonomen erkennen an, dass die Übernahme des acquis communautaire den größten Nutzen des EU-Beitritts erbrachte: Endlich bekam das Wirtschaftssystem der neuen EU-Länder einen soliden und international kompatiblen Rechtsrahmen. ${ }^{55}$ Kleinere Länder, wie die Tschechische Republik, integrierten sich durch die Europäische Union zugleich in den Prozess der wirtschaftlichen Globalisierung. ${ }^{56}$

Über die Kernelemente der Römischen Verträge hinaus bleibt die Europäische Union jedoch als ein politisches Modell umstritten. Die Spaltung geht quer durch die Lager der staatsnationalen und nationalföderativen Parteien.

Einerseits stellt die Europäische Union der sechs Gründungsmitglieder für die LiberalKonservativen (wie die tschechische ODS) kein akzeptables wirtschaftliches und soziales Modell dar, da es als zu zentralistisch und etatistisch wahrgenommen wird. Andererseits scheint die Europäische Union vielen national gesinnten und/oder linksorientierten Parteien in Ostmitteleuropa als zu liberal; so ruft die polnische national-konservative Partei Recht und Gerechtigkeit (PiS) nach der protektionistischen Rolle des Nationalstaates, somit der Stärkung der europäischen Umverteilungsmechanismen.

Die euroskeptischen Parteien haben allerdings ihre Haltung etwas moderiert, nachdem die Mitgliedschaft zur Realität geworden ist und zumal manche von ihnen ihre Vertreter im Europäischen Parlament haben: Die Sozialisierung durch Einbindung zeigt auch in diesen Parteien Wirkung. Vergleichende Analysen zeigen, dass die MOE-Staaten in ihren Ordnungsvorstellungen gespalten sind: einerseits stehen sie den euroskeptischen Ländern näher, wenn es um Liberalisierung und geringes Niveau der ,Entmarktlichung' wichtiger Lebensbereiche zum Schutz des Bürgers geht; in ihrer Unterstützung der Verteilungspolitik ähneln sie allerdings den Kohäsionsländern in der Europäischen Union. In keinem der beiden wichtigen Bereiche stehen sie den Gründungmitgliedern nahe. ${ }^{57}$ Statt die Gründungmitglieder als Modell zu betrachten, erwarten die neuen Mitgliedsländer, dass die EU-Altmitglieder ihrerseits strukturelle Anpassungen ihrer Wirtschaft durchführen. ${ }^{58}$ Diese Mischung entspricht der inneren Spaltung vieler MOE-Länder, in denen die politischen und wirtschaftlichen Eliten eine weitere Liberalisierung fordern, während die Öffentlichkeit mehrheitlich die Besitzstandwahrung vorzieht.

Radikale anti-europäische Stimmen konzentrieren sich auf die ungleiche Behandlung der neuen EU-Mitglieder und bezeichnen sie als ,weiße Sklaven“. ${ }^{59}$ Manche Analytiker glauben diese Ungleichheit werde in eine permanente Peripheriesierung einmünden. Zum festen Be-

55 Eva Klvačová: Institutional and Economic Impact of EU-Accession for the Czech Republic, in: Šikula/Hvozdíková (Hrsg.): Performance and Perspectives of the European Union as Seen by the New Member States, 2006, S. 35-60, hier S. 36.

56 Karel Zeman: The EU-Accession Effects and Impacts on the Czech Economy Efficiency, in: Šikula/Hvozdíková (Hrsg.): Performance and Perspectives of the European Union as Seen by the New Member States, 2006, S. 93-115, hier S. 110.

57 Marius Busemeyer/Christian Kellermann/Alexander Petring/Andrej Stuchlik: Europäische Verteilungskonflikte und sozialdemokratische Positionen zum Europäischen Wirtschafts- und Sozialmodel, in: Internationale Politik und Gesellschaft 4/2006, S. 138-156.

58 Rysuzard Michalski: Poland's Convergence Problem, in: Šikula/Hvozdíková (Hrsg.): Performance and Perspectives of the European Union as Seen by the New Member States, 2006, S. 116-124, hier S. 116.

59 Michał Buchowski: When Myth Becomes Reality: Polish Identities During and After EU Accession, in: Amelie Kutter/Vera Trappmann (Hrsg.): Das Erbe des Beitritts. Europäisierung in Mittel- und Osteuropa, BadenBaden 2006, S. 135-154, hier S. 149. 
standteil des nationalen Konsens in allen MOE-Staaten gehört die kritische Haltung bezüglich der Agrarpolitik der Europäischen Union. Diese wird außerhalb der Agrarlobby weitgehend als ein uneffektives, anti-marktwirtschaftliches und unfaires Instrument betrachtet, sowie als ein Beispiel der Erstarrung der Politik der westlichen Länder.

Befürchtungen bezüglich der Kosten, des institutionellen Aufwands und der Effizienz begleiten die Debatten über die meisten Sektorpolitiken. Tschechien wie die anderen Neumitglieder ist in der Frage der Vergemeinschaftung neuer Politikbereiche eher zurückhaltend. Andererseits wird auch dort immer wieder mehr EU-Solidarität eingefordert - siehe beispielsweise die Initiativen Polens im Bereich der Energiepolitik der Europäischen Union angesichts der deutsch-russischen Vereinbarung über die North Stream Pipeline.

\section{Das Argument der deutschen Hegemonie}

Der Blick der Ostblockstaaten auf die Bundesrepublik wurde in den 1950er bis 1980er Jahren als Unterfall der Ost-West Konfrontation behandelt. Die Bundesrepublik wurde als eine Triebkraft des Integrationsprozesses gesehen, die eigene ,revanchistische“ und ,expansionistische" Ziele verfolgte. ${ }^{60}$ Seit den Ostverträgen wuchs allerdings auch die Annerkennung für die außenpolitische Emanzipation der Bundesrepublik. Die Verstärkung des ,,westeuropäischen Machtzentrums des Imperialismus “ führte zu wachsenden Divergenzen in den deutsch-amerikanischen Beziehungen, die die ursprüngliche Konzeption der gleichgewichtigen euro-atlantischen Ausrichtung der Politik der Bundesrepublik gefährdete. ${ }^{61}$ Atlantiker in den MOE-Staaten befürchten auch heute noch, dass Deutschland als Spalter auftritt.

Die entscheidende Frage ist jedoch weiterhin die nach der Macht Deutschlands, nach ihrer Form und Ausübung innerhalb der Europäischen Union. Immer wieder wurde bestätigt, dass die Rolle Deutschlands zentral vor allem für die tschechische wie auch polnische Wahrnehmung der Europäischen Union ist. Die Bundesrepublik zeigte sich durchweg als ein kooperativer Partner. Die Fragen der Vergangenheit fanden zufriedenstellende Lösungen, unter anderem im Zuge der Erweiterung von NATO und Europäischer Union, die die deutsche Politik nachhaltig unterstützte. Der Versuch, die Fragen der Vergangenheit zu instrumentalisieren trieb die polnische Politik 2007 in eine (Selbst)Isolation. Die hard power Deutschlands bleibt auch nach der Wiedervereinigung relativ begrenzt und selbst ihre traditionelle soft power ist seit Anfang der 1990er Jahre geschwächt worden und konnte erst unter der großen Koalition wieder an Gewicht gewinnen. Die Öffentlichkeit nimmt heute aus allen diesen Gründen weitgehend eine gelassenere Einstellung gegenüber Deutschland ein: 62 Prozent der Polen glaubten Ende 2005, Deutschland trage zur Zusammenarbeit in Europa bei und Berlin sei neben Washington der wichtigste Partner. ${ }^{62}$ Die positive Bewertung der tschechisch-deutschen Beziehungen ist von 45 Prozent im Jahre 1996 auf 80 Prozent im Jahre 2005 gewachsen. ${ }^{63}$ Andererseits ist keine besondere politische Nähe entstanden. Das sozial-ökonomische Modell Deutschland wird in vielen MOE-Staaten nach 1989 als teuer, wenig flexibel und deswegen als kaum übertragbar angesehen. Es wird eher das skandinavische oder das angelsächsische Modell bevorzugt. ${ }^{64}$

60 История международных отношений и внешней политики СССР, 1987, Bd. 2, S. 268-269.

61 В.Н.Шенаев, М.Шмидт, Д.Е.Мельников: Федеративная республика Германии. Москва 1983, S. 373-374.

62 Meinungen der Polen über die polnisch-deutschen Beziehungen nach dem Regierungswechsel in beiden Ländern. Eine Meinungsumfrage des Institute of Public Affairs in Warschau, (Befragung vom 25-28.11.2005), S. 3 und 5, abrufbar unter: http://www.isp.org.pl/files/11811737240983198001136557801.pdf (letzter Zugriff: 10.09.2007).

63 Daniel Kunštát: Středoevropské sousedství v perspektivě veřejného mínění, in: Daniel Kunštát (Hrsg.): České veřejné mínění: výzkum a teoretické souvislosti, Praha 2006, S. 167-178, hier S. 174. 
Die Einstellung zu Deutschland fällt vor allem in den politischen Eliten sehr unterschiedlich aus: Die Abgrenzung gegenüber den europapolitischen Präferenzen Deutschlands ist vor allem in den staatsnational orientierten Parteien (in Tschechien also in der Liberal-Konservativen und bei den Kommunisten) zu einem Identifikationsthema geworden. Die tschechische ODS versuchte der deutschen „föderalistischen Politik“ entgegenzutreten und sie auszubalancieren. ${ }^{65}$ Eine viel radikalere Haltung bezog allerdings die Regierung von Lech Kaczynski, die den früheren polnischen Regierungen ,eine Vasallen-Politik gegenüber der Europäischen Union und ihren größten Mitgliedern, vor allem gegenüber Deutschland“ vorwarf. ${ }^{66}$ Unterschwellig wird die europäische Integration als ein deutsches Projekt wahrgenommen. Auch der tschechische Präsident hatte zunächst vor den Bemühungen der deutschen Ratspräsidentschaft gewarnt und später die Ergebnisse des EU-Gipfeltreffens vom Juni 2007 als einen Schritt in die falsche Richtung abgelehnt. ${ }^{67}$

\section{Schlussfolgerungen}

Die Argumente der ideologisierten Wahrnehmung der europäischen Integration aus den 1950er bis 1980er Jahren sind - außer bei der radikalen Linken - zumeist schon vor dem Jahre 1989 irrelevant geworden. Das ehemalige Bild von der Europäischen Gemeinschaft als eines Feindes ist nach dem Zerfall des Ostblocks durch das alternativlose Streben nach dem EU-Beitritt ersetzt worden. Der Beitrittsprozess und die ersten Erfahrungen der EU-Mitgliedschaft führten dann zu einem differenzierten Europabild. Heute überwiegt eine recht positive und pragmatische Sichtweise. Einzelne Themen der ehemaligen Ostblock-Propaganda werden jedoch in veränderter Form weiter diskutiert - und spiegeln auch aktuelle ideologische und kulturelle Präferenzen wider. Eine dezidierte Meinung über die Europäische Union haben vor allem die Vertreter von euroskeptischen Konzepten unter den Eliten.

Ostmitteleuropa zeigte eine historisch beispiellose Kongruenz der außenpolitischen Orientierung nach dem Jahr 1989: die Region, die in der Geschichte immer wieder Spaltungen, innere Konflikte und äußere Vorherrschaft erfahren hat, hat sich beim Streben nach der Integration in den Westen einig gezeigt. In ihrer Alternativlosigkeit erinnert diese Ausrichtung an Konrad Adenauers außenpolitischen Ansatz in der Bundesrepublik - inklusive der Vernachlässigung der Dimensionen der Außenpolitik, die über den euro-atlantischen Rahmen hinausgingen. Deutschland ist vor allem für die tschechische wie auch die polnische Europapolitik derjenige Partner, mit dem die häufigsten und beständigsten Kontakte bestehen.

Im Prozess der schrittweisen Differenzierung des Europabildes haben die Römischen Verträge eine fundamentale Bedeutung: Denn sie umreißen den Kernbestand des Grundkonsens der maßgeblichen politischen Kräfte, der den EU-Beitritt ermöglichte. Über die Römischen Verträge hinaus - also in Bezug auf die Entwicklungen vom Maastrichter Vertrag bis zum Verfassungsvertrag für Europa - ist die politische Szene vor allem in Tschechien und Polen weitgehend gespalten. Fällt die ,Rückkehr nach Europa“ mit einer ,Rückkehr zum Nationalstaat"

64 Anna Kadeřábková: Challenges for the Competitiveness of the Czech Republic Within the EU, in: Šikula/ Hvozdíková (Hrsg.): Performance and Perspectives of the European Union as Seen by the New Member States, 2006, S. 26-34, hier S. 30.

65 Vlastimil Havlík: Česká republika, in: Havlík/Kaniok (Hrsg.): Euroskepticismus a země střední a východní Evropy, 2006, S. 61-82, hier S. 68.

66 Pior Buras: Gelingt Europa gemeinsam? Polens Europapolitik und die deutsche EU-Ratspräsidentschaft, Konrad-Adenauer-Stiftung, Warschau 2005, S. 6, abrufbar unter: http://www.kas.de/db_files/dokumene/7_dokume nt_dok_pdf_11062_1.pdf (letzter Zugriff: 10.09.2007).

67 Václav Klaus: EU in der Zeit nach der deutschen EU-Präsidentschaft, Rede an der Festakademie des HerbertBatliner-Europainstituts, Salzburg, 28.07.2007, abrufbar unter: http://www.klaus.cz/klaus2/asp/clanek.asp?id= QWmp6ua0t5rc (letzter Zugriff: 10.09.2007). 
zusammen? In der Politik der staatsnational orientierten Parteien scheint es so zu sein. Sich von Deutschland und Deutschlands Europapolitik abzugrenzen spielt dabei eine wichtige Rolle.

Die Öffentlichkeit steht dem Bild der Europäischen Union als einer Wohlfahrts- und Friedensgemeinschaft am nächsten. In allen gesellschaftlichen Schichten ist die Europäische Union als Wirtschafts- und Stabilitätsgemeinschaft akzeptiert. Vor allem ihr Bild als Gemeinschaft der postmaterialistischen Werte und als Sicherheitsgemeinschaft spaltet aber die politischen Eliten.

Es ist richtig, dass der EU-Beitritt keine parteipolitische Polarisierung im Sinne eines langfristigen cleavage bewirkte, sondern umgekehrt die schrittweise Europäisierung vorantreibt - auch die der euroskeptischen Parteien. ${ }^{68}$ Der disziplinierende Effekt des Beitritts ist allerdings weggefallen. Die europapolitische Polarisierung der Eliten und der enge Rahmen des gesellschaftlichen Konsens erschweren die Profilierung der Europapolitik und ihre effektive Ausübung. Auch aus diesem Grund ist die Bilanz der tschechischen Aktivitäten bei den EU-Gipfeln und Ratssitzungen ,,als bescheiden zu bewerten“ 69

Inwieweit die Polarisierung auch ein Nachhall der ideologisierten Präsentation der europäischen Integration in Zeiten des Kalten Krieges ist, dürfte ein Thema für die weitere Forschung sein. Vielfach scheint es vor allem das damalige Verständnis der Politik als eines Kampfes zwischen unvereinbaren Gegensätze zu sein, das heute den Elitendiskurs über die Europäische Union bestimmt.

68 Michael Baun/Jakub Dürr/Dan Marek/Pavel Šaradín: The Europeanization of Czech Politics: The Political Parties and the EU Referendum, in: Journal of Common Market Studies 2/2006, S. 249-280, hier S. 274.

69 Kristína Larischová: Tschechien, in: Werner Weidenfeld/Wolfgang Wessels (Hrsg.): Jahrbuch der europäischen Integration 2006, Baden-Baden 2007, S. 409-412, hier S. 412. 